\title{
CONCEPÇÕES E PRÁTICAS DO AGENTE COMUNITÁRIO NA ATENÇÃO À SAÚDE DO HOMEM
}

\author{
CONCEPTIONS AND PRATICES OF COMMUNITARY \\ HEALTH CARE AGENTS
}

\author{
Cleusimara Tumasz*, Erildo Vicente Muller** \\ * Enfermeira, especialista em saúde coletiva: gestão de serviços de saúde e saúde da \\ família - UEPG \\ ** Docente do departamento de enfermagem e saúde pública - UEPG
}

Recebido para publicação em 06/04/2010

Aceito para publicação em 14/07/2010

\section{RESUMO}

Trata-se de um estudo que procura analisar o atual conhecimento e as práticas de trabalho do agente comunitário de saúde prestado ao homem, demonstrando a necessidade de um aperfeiçoamento técnico e atualizações que promovam um atendimento diferenciado e atrativo, no que se refere à atenção primária a este segmento da população. A metodologia empregada foi do tipo exploratória/ descritiva e a pesquisa realizada no município de São João do Triunfo - Pr, com 25 agentes comunitários de saúde. Os dados foram coletados por meio de formulário com questões abertas e fechadas. Para análise das informações utilizou-se estatística descritiva e análise de categorias nas questões abertas. Observou-se que $76 \%$ dos entrevistados trabalham como agentes de saúde há mais de 4 anos e $84 \%$ não participaram de aperfeiçoamento em saúde do homem. Concluiu-se que os ACS necessitam de maior preparo no que se refere à saúde do homem, por se tratarem de profissionais diretamente envolvidos no trabalho de orientação e prevenção de agravos.

Palavras-Chave: Agente comunitário de saúde. Saúde do homem. Atenção primária.

\begin{abstract}
This study analyzes current knowledge and work practices of agents involved with Community Health Care (CHC) provided to men in order to demonstrate the need of technical update and in-service training to promote a differentiated and attractive service in relation to primary care given to this segment of the population. An exploratory/descriptive research was conducted in São João do Triunfo - Pr with 25 community health workers. Data were collected using a questionnaire with open and closed questions. The data analysis included statistical description and category analysis of the open questions. It was observed that $76 \%$ of the respondents work as community health workers for over 4 years; and $84 \%$ did not participate in any in-service training about men's health. It was concluded that the agents of the CHC
\end{abstract}


need better preparation in relation to men's health as they are professionals directly involved in guidance work and prevention of injuries.

Keywords: Community Health Agents. Men's Health. Primary Attention.

\section{Introdução}

No Brasil, segundo dados do Instituto Brasileiro de Geografia e Estatística (IBGE, 2010), a população masculina é da ordem de $48,9 \%$ e a população feminina $50,8 \%$. Ainda de acordo com o IBGE, a expectativa de vida atual dos homens brasileiros é de 69,4 anos, enquanto para as mulheres, encontra-se em 77 anos.

Estudos comparativos entre homens e mulheres têm comprovado o fato de que os homens são mais vulneráveis às doenças, descuidam de sua saúde e devido a isso, morrem mais precocemente que as mulheres. A mortalidade é maior no sexo masculino em todas as faixas etárias, podendo este dado estar relacionado a fatores genéticos, emocionais ou ainda ao estilo de vida, que mostra-se diferente entre os dois sexos (PEREIRA, 2001).

Acredita-se que a alta taxa de morbimortalidade bem como a maior vulnerabilidade deve-se a baixa procura dos homens pelos serviços de atenção primária, que são direcionados principalmente à prevenção de doenças, recorrendo ao sistema de saúde tardiamente, tendo como conseqüência problemas de saúde mais complexos e com maior custo de tratamento (Figueiredo, 2005).

Com o objetivo de orientar e aperfeiçoar as ações e serviços de saúde direcionados à população masculina, o Ministério da Saúde implantou em agosto de 2008, a PNAISH (Política Nacional de Atenção Integral à Saúde do Homem), que visa a garantia de integralidade e equidade, primando pela humanização da atenção a saúde deste segmento (BRASIL, 2008).

Segundo o documento que especifica os princípios e diretrizes da nova política, faz-se necessário a aplicação de ações intersetoriais e multiprofissionais organizadas de modo a acolher e fazer com que o homem sinta-se parte integrante dos serviços públicos de saúde, incentivando a busca pelos serviços de prevenção e tratamento precoce, disponíveis nas redes básicas de saúde, consideradas a porta de entrada do SUS (BRASIL, 2008).

A atenção primária atualmente é caracterizada como um serviço voltado para as mulheres (FIGUEIREDO, 2005), reproduzindo desigualdades no que se refere ao funcionamento dos serviços e nos desempenhos dos profissionais, que demonstram a ausência de um olhar diferenciado ao prestar atendimento aos gêneros feminino e masculino, fato este que impossibilita uma assistência equânime, de acordo com as especificidades que requer o trato da saúde dos homens (SCHRAIBER et al., 2010).

Os profissionais de saúde devem estar sensibilizados para promover efetivamente as mudanças de paradigmas, referentes à percepção da população masculina em relação ao cuidado com sua saúde, atuando no desestímulo ao comportamento intempestivo, que compromete o acesso aos serviços e a adesão ao tratamento, sobretudo devido à crença na invulnerabilidade do homem (BRASIL, 2010; CARRARA et al., 2009; GOMES; NASCIMENTO, 2006; GOMES, 2003).

Os agentes comunitários de saúde (ACS) vêm se constituindo, nesses programas, como segmento efetivo do trabalho em saúde, representando novos atores no cenário da assistência, que se apresenta não apenas como suporte para gerar determinadas ações em saúde, mas também, como peça essencial na organização da assistência (SILVA; DALMASO, 2002).

De acordo com Silva e Dalmaso (2002), dentre as competências do ACS destaca-se a promoção da saúde, através do desenvolvimento de trabalho educativo, do estímulo à participação comunitária e do trabalho intersetorial; ações que favorecem um atendimento integral e de qualidade aos usuários do sistema de saúde, sendo assim de extrema necessidade sua atuação no cuidado a população masculina.

Sabe-se que o homem tem procurado os serviços de saúde tardiamente, e que a prevenção e ou detecção precoce de problemas de saúde tem sido 
negligenciada pela população masculina. Uma vez que, o Ministério da Saúde, através da implantação de uma nova política, busca incentivar o cuidado com a saúde pelos homens, sendo um de seus objetivos tornar a atenção básica mais atrativa e acessível, torna-se fundamental conhecer as concepções dos profissionais de saúde no que concerne ao atendimento ao homem (BRASIL, 2008).

Por vivenciar os problemas na comunidade em que desempenha sua prática de trabalho, o ACS figura como importante elo de interlocução entre a equipe e o usuário, na produção do cuidado, viabilizando, de acordo com sua competência, a melhoria da assistência, contribuindo significativamente na aplicação de políticas públicas (SILVA; DALMASO, 2002). Dessa forma, estudos da temática, ainda que parciais e localizados, são importantes para essa discussão e para contribuir na efetivação dessa nova proposta de abordagem à saúde dos homens.

Nesse contexto o presente estudo teve como objetivo verificar as concepções que predominam entre os Agentes Comunitários de Saúde no que se refere a saúde do homem, bem como identificar as dificuldades e limitações vivenciadas pelos ACS em sua prática no trabalho com a população feminina.

\section{Materiais e métodos}

Optou-se pelo estudo exploratório descritivo, por entender-se que esse método permite analisar o conhecimento da população estudada a respeito da temática pesquisada. $\mathrm{O}$ estudo foi realizado com Agentes Comunitários de Saúde (ACS), integrantes de equipes da Estratégia Saúde da Família (ESF) na cidade de São João do Triunfo - PR. Foram entrevistados 25 indivíduos, perfazendo o total de trabalhadores dessa categoria no município.

A entrevista deu-se por meio de formulário, com perguntas abertas e fechadas que levou em conta as variáveis: idade, sexo, escolaridade, tempo de atuação na área, bem como concepções do ACS sobre a abordagem da saúde do homem em sua prática de trabalho, além de variáveis que possibilitem estimar o conhecimento do ACS em relação às suas atribuições na atenção à população masculina, as dificuldades em relação ao assunto e as queixas mais freqüentes feitas pelos homens em sua vivência de trabalho. Utilizou-se estatística descritiva, através de planilha montada no programa da Microsoft Excel versão 2002 e análise de categorias nas questões abertas.

A aplicação do instrumento de pesquisa foi efetuada pelo pesquisador, mediante assinatura do Termo de Consentimento Livre e Esclarecido (TCLE), após a aprovação do projeto de pesquisa pelo Comitê de Ética em Pesquisa da Universidade Estadual de Ponta Grossa (UEPG).

\section{Resultados e discussão}

A pesquisa aponta que $84 \%$ dos ACS participantes são do sexo feminino e $72 \%$ são adultos jovens com idade acima de 25 anos. Quanto à variável escolaridade, $60 \%$ afirmam ter 12 ou mais anos de estudo, enquanto apenas $4 \%$ referem ter 5 a 8 anos de estudo. $\mathrm{O}$ dado demonstra a necessidade desses profissionais em expandir seus conhecimentos. Essa crescente escolarização do ACS pode ser observada em outros estudos, e é atribuída à busca de uma projeção social e profissional, uma vez que grande parte dos ACS é proveniente de camadas populares, e como tais, almejam melhores condições de vida e de trabalho (MOTA; DAVID, 2010).

A criação da profissão Agente Comunitário de Saúde é recente e foi efetivada por meio da lei $\mathrm{n}^{\mathrm{o}} 10.507 / 02$, que estabelece os requisitos para atuar na função, dentre eles: residir na comunidade onde irá trabalhar, ter concluído o ensino fundamental e haver concluído com aproveitamento o curso de qualificação básica para a formação de ACS (BRASIL, 2002). Anteriormente era necessário apenas saber ler e escrever (BRASIL, 1997). Em 2004, o governo estabeleceu uma diretriz onde constitui a necessidade de formação técnica aos ACS e reafirma a intenção de associar educação profissional à elevação de escolaridade (BRASIL, 2004).

O tempo de atuação na profissão de Agente Comunitário de Saúde é de acima de quatro anos para a grande maioria dos participantes, sendo 
que apenas $24 \%$ trabalham há menos tempo. De acordo com os dados obtidos, $84 \%$ dos ACS nunca participaram de cursos, palestras ou seminários na área da saúde que tenha em seu contexto o tema: Saúde do Homem. Tal indicador mostra que apesar da experiência e do tempo em que atuam na área, estes profissionais apresentam dificuldades em obter informações técnicas e direcionadas que venham a facilitar e tornar efetivas as ações voltadas à população masculina.

Para que se possa falar em prevenção, ou em atenuar o progresso das doenças é preciso adquirir conhecimento sobre as causas ou fatores predisponentes que influenciam direta ou indiretamente na aquisição ou desenvolvimento de problemas de saúde (GOMES et al., 2008).

Segundo o documento que rege a Política Nacional de Atenção Integral a Saúde do Homem (Brasil, 2008), o fortalecimento das ações, serviços em redes e cuidados com a saúde do homem, se dará de forma alinhada à Política Nacional de Atenção Básica - porta de entrada do Sistema Único de Saúde. O ACS, como membro da equipe de Estratégia Saúde da Família (ESF), é um dos operantes que deve promover a realização dos objetivos dessa nova política, que busca estimular o autocuidado masculino, garantindo a promoção da saúde e a prevenção de agravos evitáveis.

As inferências deste estudo revelaram que $60 \%$ dos ACS não se consideram preparados para orientar aos homens de sua área de atuação, sobre os cuidados com sua saúde, atribuindo o despreparo ao fato de não ter recebido treinamento específico para tal:

"faltam mais informações, como fazer a abordagem."

"sei um pouco, tenho pouca informação."

"simplesmente penso que não tenho total conhecimento sobre o assunto."

"não totalmente preparado, pois nunca tivemos um estudo mais detalhado."

Um dos ACS entrevistados afirma que obtém conhecimento sobre saúde do homem através de panfletos enviados pelo Ministério da Saúde (MS):

"sempre que podemos, lemos panfletos que falam sobre o assunto e levamos até eles."
Essa fala reforça, de certa forma, a necessidade de um maior aprofundamento sobre as peculiaridades do tema em questão, pois os referidos panfletos apresentam informações em tópicos, resumidas, que são produtivas a partir do momento em que chegam ao público alvo, ou seja, aos homens, que por sua vez, tem seu interesse despertado, buscando assim, maiores e mais completas informações junto aos profissionais de saúde. Para tanto, esses profissionais devem estar preparados para dar continuidade ao trabalho iniciado através de panfletagem e divulgações em meios de comunicação, acrescentando e esclarecendo as eventuais dúvidas ou anseios dos usuários. Assim, no campo da atenção primária, é de extrema importância que as informações sejam seguras e abrangentes.

Os dados obtidos mostram que $96 \%$ dos entrevistados têm conhecimento de que os homens buscam os serviços de saúde tardiamente, não se preocupando com a prevenção. Grande parte dos ACS revelou conhecer as principais causas de morte entre os homens adultos e o mesmo índice é apontado ao se questionar sobre o objetivo da Política Nacional de Atenção Integral a Saúde do Homem, com $84 \%$ dos entrevistados demonstrando conhecê-lo.

O câncer de próstata e neoplasias, além das práticas preventivas relacionadas a esses agravos, está dentre os temas nos quais os Agentes Comunitários demonstram interesse em obter mais conhecimento (Tabela 1), a fim de facilitar o trabalho de orientação durante as visitas domiciliares.

Tabela 1 - Temas pelos quais os ACS referem interesse em obter mais conhecimento. São João do Triunfo, 2010 .

\begin{tabular}{ll}
\hline Categoria & $\boldsymbol{N}(\boldsymbol{\%})$ \\
\hline Câncer de Próstata, outras neoplasias e exames & \\
preventivos ou de detecção precoce & $24(60)$ \\
Riscos do uso inadequado de agrotóxicos & $09(22,5)$ \\
Problemas relacionados à pele & $04(10)$ \\
Hipertensão e problemas cardíacos & $03(7,5)$ \\
Total & $\mathbf{4 0}(\mathbf{1 0 0})$ \\
\hline
\end{tabular}

Fonte: Formulário Aplicado.

A característica de realizar cuidados e exames preventivos não é comum aos homens, pois procuram os serviços de saúde, na maioria das vezes, 
quando já apresentam doença manifesta, valorizando mais as práticas curativas (SCHRAIBER et al., 2010).

No Brasil, o câncer de próstata tem se mostrado um grave problema de saúde pública. Segundo o Instituto Nacional de Câncer (INCA), o câncer de próstata possui altas taxas de incidência e mortalidade, tornando-o a segunda neoplasia mais comum entre a população masculina, sendo superado apenas pelo câncer de pele não-melanoma (INCA, 2010).

Nesse contexto, o interesse apontado pelos agentes de saúde é de grande valia, pois a prevenção e o diagnóstico precoce é uma importante medida para evitar o surgimento ou o progresso do câncer de próstata (INCA, 2010), podendo ser classificada como prevenção primária aquela que faz referência a fatores causais ou predisponentes, em doenças cujas causas são conhecidas; e prevenção secundária, aquela onde as ações voltam-se ao diagnóstico e tratamento precoce de doenças que por sua vez tenham causas menos conhecidas (GOMES et al., 2008).

Dentre os temas relacionados como necessários de aperfeiçoamento, destaca-se o que se refere ao uso inadequado de agrotóxicos. O problema sugerido pelos ACS parece ser comum especialmente em municípios de pequeno porte, onde a agricultura familiar ou de subsistência é predominante, sendo a população masculina grandemente afetada.

Ao ser indagado a respeito das queixas mais freqüentes vivenciadas em seu trabalho pelos homens, os agentes apontam um índice elevado de queixas referentes à demora no atendimento, funcionamento dos serviços, além de falta de tempo e horários flexíveis nas unidades de saúde (Tabela 2). Na tentativa de justificar a baixa procura pela atenção primária, o gênero masculino utiliza-se principalmente de argumentos relacionados ao trabalho e conseqüente falta de tempo, como relatada a literatura (GOMES et al., 2007).
Tabela 2 - Queixas mais frequentes vivenciadas pelos ACS realizadas por pacientes do sexo masculino. João do Triunfo, 2010.

\begin{tabular}{ll}
\hline Categoria & $\boldsymbol{N}(\%)$ \\
\hline Demora no atendimento & $14(41,18)$ \\
Indisponibilidade e mau funcionamento dos & $13(38,24)$ \\
serviços & $04(11,76)$ \\
Falta de tempo para cuidar da saúde & $03(8,82)$ \\
Necessidade de horários flexíveis & $\mathbf{3 4}(\mathbf{1 0 0})$ \\
Total &
\end{tabular}

Fonte: Formulário Aplicado.

\section{Considerações finais}

A saúde do homem deve ser abordada de forma singular, considerando as barreiras e preconceitos que existem e são muitas vezes conseqüência da ideologia hegemônica de masculinidade, que reflete no comportamento intempestivo, na crença de que são invulneráveis às doenças e até mesmo no "apoderamento" que se atribui ao sujeito homem por eles próprios. Diversos estudos (SCHRAIBER et al., 2010; CARRARA et al., 2009; GOMES, 2003; GOMES et al., 2007) corroboram com a afirmativa de que tal comportamento masculino pode dificultar a adoção de hábitos e convicções saudáveis.

A partir da percepção de que os homens estão desatentos à prevenção de doenças, bem como da detecção precoce das mesmas, torna-se imprescindível alcançá-los através da informação consistente, tornando atrativos e usuais os serviços de atenção básica e ainda desestimulando o pensamento de que cuidar da saúde é um sinal de fragilidade.

O presente estudo mostrou que grande parte dos agentes de saúde aponta para uma carência de conhecimento no que concerne à saúde do homem e suas especificidades, demonstrando interesse em aperfeiçoar-se, uma vez que sabem da resistência da maioria dos homens em relação ao autocuidado.

Assim, para que os trabalhadores de saúde, sobretudo os ACS, que representam a ligação direta entre o serviço e a população, possam despertar no segmento masculino o hábito de cuidar preventivamente da saúde, é preciso preparar-se para tal. Somente o conhecimento promove com eficácia a prevenção. 


\section{REFERÊNCIAS}

BRASIL. Ministério da Educação. Referencial curricular para curso técnico de agente comunitário de saúde: área profissional saúde. Acesso em 30 outubro 2010. Disponível em: <http://iah.iec.pa.gov.br/iah/fulltext/pc/monografias/ms/ sp/referencial_Curricular_ACS.pdf $>$

Presidência da República. Subchefia de Assuntos Jurídicos. Lei n. ${ }^{\circ} 10.507$, de 10 de julho de 2002. Cria a profissão de agente comunitário de saúde e dá outras providências. Diário Oficial da União. Acesso em: 30 outubro 2010. Disponível em: <http://dtr2004.saude.gov.br/dab/docs/ legislacao/lei10507_10_07_02.pdf $>$

. Ministério da Saúde. Política nacional de atenção integral à saúde do homem (princípios e diretrizes). Acesso em: 15 mar. 2010. Disponível em: <http://dtr2001.saude.gov. br/sas/PORTARIAS/ Port 2008/PT-09-CONS.pdf>

. Ministro de Estado da Saúde. Portaria n. ${ }^{\circ}$ 1.886/GM, de 18 de dezembro de 1997. Aprova as normas e diretrizes do programa de agentes comunitários de saúde e do Programa de Saúde da Família. Diário Oficial da República Federativa do Brasil. Acesso em: 30 outubro 2010. Disponível em: <www. saude.sc.gov.br/PSF/PORTARIAS/Portaria\%20n1886\%20-\%20original\%2018dez1997.doc>

CARRARA, S; RUSSO, J. A; FARO, L. A política de atenção à saúde do homem no Brasil: os paradoxos da medicalização do corpo masculino. Physis Rev Saúde Coletiva, v.19, n.3, p.659-78, 2009.

FIGUEIREDO, W. Assistência à saúde dos homens: um desafio para os serviços de atenção primária. Rev. C. S. Col., v.10, n.1, p.105-9, 2005.

GOMES, R. Sexualidade masculina e saúde do homem: proposta para uma discussão. $\operatorname{Rev} \mathbf{C ~ S ~ C o l ; ~ v . 8 , ~ n . 3 , ~ p . 8 2 5 - ~}$ $29,2003$.

.; NASCIMENTO, E. F. A produção do conhecimento da saúde pública sobre a relação homem-saúde: uma revisão bibliográfica. Cad. Saúde Pública, v.22, n.5, p.901-11, 2006.

; $\quad$; $\quad$;ARAÚJO, F. C. Por que os homens buscam menos os serviços de saúde do que as mulheres? As explicações de homens com baixa escolaridade e homens com ensino superior. Cad. Saúde Pública, v.23, n.3, p.565-74, 2007.

; REBEllo, L. E. F. S; ARAÚJO, F. C; NASCIMENTO, E. F. A prevenção do câncer de próstata: uma revisão de literatura. Rev C S Col., v.13, n.1, p.235-46, 2008.

IBGE. Instituto Brasileiro de Geografia e Estatística. Censos demográficos e contagem populacional para os anos intercensitários. Acesso em: 12 dezembro 2010. Disponível em: $<$ http://www.ibge.gov.br/censo 2010/>
INCA- Instituto Nacional de Câncer. Prevenção do câncer de próstata. Acesso em: 30 novembro 2010. Disponível em: $<$ http://www.inca.gov.br/impress\%E3o $>$

MOTA, R. R. A; DAVID, H. M. S. L. A crescente escolarização do agente comunitário de saúde: uma indução do processo de trabalho? Trab. Educ. Saúde, v.8, n.2, p.229-48, 2010.

PEREIRA, M. G. Epidemiologia teoria e prática. Rio de Janeiro: Guanabara Koogan, 2001.

SCHRAIBER, L. B; FIGUEIREDO, W. S; GOMES, R; COUTO, M. T; PINHEIRO, T. F; MACHIN, R; SILVA, G. S. N; VALENÇA, O. Necessidades de saúde e masculinidades: atenção primária no cuidado aos homens. Cad. Saúde Pública, v.26, n.5, p.961-70, 2010.

SILVA, J. A; DALMASO, A. S. W. Agente comunitário de saúde: o ser, o saber, o fazer. Rio de Janeiro: Fiocruz; 2002. 


\section{ANEXO A - FORMULÁRIO}

1- Iniciais do Nome:

Idade:

Sexo: ( ) Masculino

( ) Feminino

2- Escolaridade:

( ) 1 a 4 anos de estudo

() 5 a 8 anos de estudo

( ) 9 a 11 anos de estudo

( ) 12 ou mais anos de estudo

3- Tempo de atuação na profissão de Agente Comunitário de Saúde:
( ) 1 mês a 6 meses
( ) 6 meses a 1 ano
() 1ano a 2 anos
( ) 2 anos a 3 anos
( ) 3 anos a 4 anos
( ) 4 anos ou mais

4- Você já participou de cursos, palestras ou seminários na área da saúde que tenha em seu contexto o tema: Saúde do Homem? Se sim, há quanto tempo?
( ) $\mathrm{Sim}$
( ) Não

Há quanto tempo?

( ) Nos últimos 6 meses.

( ) Há menos de 1 ano.

( ) Há mais de 1 ano.

( ) Há mais de 2 anos.

5- De acordo com o seu conhecimento, você considera-se preparado para orientar aos homens de sua área de atuação, sobre os cuidados com sua saúde? Justifique sua resposta:
( ) $\mathrm{Sim}$
( ) Não

Justificativa:

6- Assinale as afirmativas corretas em relação a Saúde do Homem:

( ) Os homens buscam os serviços de saúde tardiamente, não preocupando-se com a prevenção, tendo como conseqüência o agravamento de seus problemas de saúde.

( ) Os homens adoecem e morrem menos que as mulheres, porque são geneticamente mais fortes.

( ) As principais causas de morte entre os homens adultos são: Causas Externas ( acidentes de trânsito, lesões autoprovocadas, agressões), Problemas do Aparelho Circulatório ( problemas cardíacos, hipertensão...) e Tumores.

( ) O câncer de próstata é uma neoplasia que geralmente apresenta evolução muito lenta, desse modo a mortalidade pó essa doença pode ser evitada quando se faz o diagnóstico e tratamento precoce, através de exames preventivos.

( ) A Política Nacional de Atenção Integral a Saúde do Homem (PNAISH) tem o objetivo de tornar obrigatório o tratamento hospitalar dos homens hipertensos.

7- Cite três assuntos relacionados à Saúde do Homem nos quais você gostaria de obter mais conhecimento, facilitando o seu trabalho de orientação nas visitas domiciliares.

8- Na sua prática de trabalho, quais as queixas mais frequentes mencionadas pelos moradores do sexo masculino em relação ao serviço de saúde? 